\title{
The Prevalence of Mind-Body Dualism in Early China
}

\author{
Edward Slingerland, ${ }^{\mathrm{a}}$ Maciej Chudek ${ }^{\mathrm{b}}$ \\ ${ }^{a}$ Department of Asian Studies, University of British Columbia \\ ${ }^{\mathrm{b}}$ Department of Psychology, University of British Columbia
}

Received 5 November 2010; received in revised form 25 January 2011; accepted 28 January 2011

\begin{abstract}
We present the first large-scale, quantitative examination of mind and body concepts in a set of historical sources by measuring the predictions of folk mind-body dualism against the surviving textual corpus of pre-Qin (pre-221 BCE) China. Our textual analysis found clear patterns in the historically evolving reference of the word xin (heart/heart-mind): It alone of the bodily organs was regularly contrasted with the physical body, and during the Warring-States period became ever less associated with emotions and increasingly portrayed as the unique locus of "higher" cognitive abilities. We interpret this as a semantic shift toward a shared cognitive bias in response to a vast and 1 rapid expansion of literacy. Our study helps test the proposed universality of folk dualism, adds a new quantitative approach to the methods used in the humanities, and opens up a new and valuable data source for cognitive scientists: The record of dead minds.
\end{abstract}

Keywords: Mind-body dualism; Folk cognition; Comparative psychology; Chinese thought; Textual analysis

\section{Introduction}

The idea that mind-body dualism is a folk cognitive universal is widespread in the cognitive sciences. This position is perhaps best exemplified by Paul Bloom's argument that the apparent early onset on mind-body dualism in infant development, its automaticity, and its apparent cross-cultural universality suggests that we are "natural Cartesians" (Bloom, 2004: xii).

Bloom's claim for a universal folk Cartesianism might be characterized as "strong folk dualism" (SFD), in that it involves a scalpel-sharp, clear divide between a physical, mechanistic body and a disembodied, ontologically distinct mind encompassing abstract

Correspondence should be sent to Edward Slingerland, Department of Asian Studies, University of British Columbia, Asian Centre, 403-1871 West Mal, Vancouver, BC V6T 1Z2, Canada. E-mail: edward.slingerland@ ubc.ca

\begin{tabular}{|c|c|c|c|c|c|c|c|c|}
\hline \begin{tabular}{|l|l}
$\mathrm{C}$ & $\mathbf{0}$ \\
\end{tabular} & & $\mathbf{S}$ & 1 & 1 & \begin{tabular}{l|l}
8 & 6
\end{tabular} & & \multicolumn{2}{|l|}{ Dispatch: 18.511 } \\
\hline Journ: & Name & & Mant & uscript & No. & 1 & Author Receivec & \\
\hline
\end{tabular}


cognition, intentions, emotions, and personal identity-the "ghost in the machine.' Strong dualism has been challenged by recent work suggesting that "weak folk dualism" (WFD), whereby mind-stuff and body-stuff overlap and interact, is our cognitive default (Hodge, 2008). When reasoning about topics such as spirit possession or the afterlife, study participants have intuitions about which capacities clearly go with "the mind" (abstract thoughts and personal identity); which clearly go with "the body" (physiological functions); and which are intermediate capacities, such as appetites and habits, that straddle body and mind (Cohen, 2007; Cohen \& Barrett, 2008). Historical data also supports WFD: Many, if not most, philosophical and religious traditions throughout history have postulated multi-partite models of the self's capacities that only roughly coordinate with Cartesian mind-body dualism. ${ }^{1}$ WFD predicts that, while it is not characterized by a perfectly consistent and mutually exclusive divide between mind and body, human cognition will tend to cluster person-concepts around these attractors, with only a rare minority of concepts conflating the two in special cultural or pragmatic contexts. According to WFD, Descartes' austere mind-body substance dualism represents an intellectually rigorous, albeit ultimately counter-intuitive, working out of this folk intuition.

Both SFD and WFD predict that a universal cognitive distinction between body and mind will shape and constrain the evolution of language and culture, producing a clear, crosscultural trend toward contrasting mind-body concepts. The absence of such a trend, while it would not indisputably falsify either form of folk dualism, would constitute a challenge for both. SFD additionally predicts that all aspects of cognition-including emotions, desires, and habits - should move in lockstep with higher cognitive abilities (abstract thought, decision-making) and be sharply contrasted with the body, whereas WFD allows for intermediate capacities with varying links to the body.

A third position, mind-body holism $(\mathrm{MBH})$, rejects both WFD and SFD, arguing that mind-body dualism in any form is characteristic only of European-descended cultures, and therefore does not reflect an innate cognitive universal. For instance, Wierzbicka, 2006 argues against an exact equivalent to the English word mind in other cultures/languages:

The other main part of the person...is conceived of differently in different cultures. Roughly speaking, the (contemporary) English word mind presents this part as primarily an organ of thinking and knowing, whereas the Russian word dusa, the Japanese word kokoro $^{2}$ and the Korean word maum link it in various ways with feeling, wanting, and choosing between what is "good"' and what is "bad"' (165)

In addition at least one language, classical Chinese, lacks a single word corresponding to the English body, which could alternately be rendered as shen 身, $t i$ 體, or xing 形. Though both forms of dualism accommodate the possibility that languages will not necessarily divide up this semantic range the same way as do the English words mind and body, the claim that dualism is a cognitive universal suggests that all languages will contain semantic divisions that ultimately boil down to the mental and the physical. While many critiques of innate mind-body dualism assume that this is not the case, the issue has not been rigorously empirically explored. 
This study responds to this gap by presenting the first large-scale, quantitative examination of concepts of mind and body in a set of historical sources, measuring the predictions of folk dualism against the surviving textual corpus of pre-Qin (pre-221 BCE) China. This study both pioneers a quantitative approach to the analysis of historical texts and allows cognitive science to tap into a valuable and hitherto underutilized source of data.

Pre-Qin China represents a particularly important test-case for folk dualism because of longstanding and widespread claims that mind-body dualism is entirely foreign to China-or "the East"' more generally (Granet, 1934; Jullien, 2007; Lévy-Bruhl, 1922; Rosemont \& Ames, 2009). Moreover, the relative historical continuity and remarkable geographical scope of the Chinese cultural sphere, which was fundamentally shaped during the pre-Qin period, makes it an important data point for any claims concerning human cognitive universals. Finally, the pre-Qin period we examined represent a unique stage in the evolution of Chinese written communication where literacy began to spread from the specialized, exclusive needs of a small priestly class to a far larger and more diverse literate population (Lewis, 1999). If this broader population did share cognitive biases, this is precisely the period when we would expect the semantic scope of Chinese characters to evolve to reflect those biases.

Supporters of MBH in early China (Geaney, 2002; Jullien, 2007; Yu, 2007) claim that xin 心, the term in classical Chinese typically translated as mind, also denotes a physical organ (the graph 心, in its original form, clearly depicts the heart), and that, in addition to the cognitive capacities associated with mind, xin also serves as the locus of emotions, desires, and imaginative capacities. Though moderate versions of this claim are consistent with weak dualism, proponents of $\mathrm{MBH}$ typically make the stronger claim that there exists no qualitative distinction at all between anything we could call mind and the physical body or other organs of the body. For instance, Yu (2007) (citing approvingly several contemporary scholars of Chinese thought) claims that "the heart' and in their Western senses, are conceptualized in ancient Chinese philosophy as being one, the xin 'heart,' which houses thoughts and feelings, ideas and emotions"'; Jullien (2007) similarly declares that, because the Chinese saw what we would call body, soul, and mind as nothing more than points along a continuous, constantly transforming spectrum of energy, "no dualism is possible."

In order to test these sorts of claims, we drew a large $(n=620)$ random sample of passages containing the word xin in both received and archeological texts from pre-Qin China. These texts can be roughly classified into three periods: Pre Warring States (c. 1500 to c. 475 BCE), Early Warring States (late fifth to mid-fourth century BCE), and Late Warring States (mid-fourth century BCE-221 BCE). We aimed to determine:

- whether these passages explicitly contrasted xin with the physical body and its other physical organs [contrary to MBH and consistent with both SFD and WFD];

- the relative frequency of xin referring to a (a) physical organ, (b) a locus of lower cognition (feelings, emotions), and (c) a locus of higher cognition (thought, reasoning, decision-making) [approximately equal frequencies would be most consistent with 
$\mathrm{MBH}$; predominant but equal reference to (b) and (c) would favor SFD; predominant reference to both (b) and (c) with significantly more (c), would favor WFD];

- whether these patterns changed over time.

\section{Methods}

We extracted passages containing xin from an on-line database of the entire received pre-Qin corpus, ${ }^{3}$ as well as a database of a cache of recently discovered pre-Qin archeological texts. ${ }^{4}$ The result was 1,321 passages, automatically chunked into standard textual units by the search engine. The first author and three hypothesis-blind coders randomly sampled 60 passages and inductively developed a set of 29 dichotomous codes to classify its usage (see Table S1). Of these, two main categories of codes bear directly on the analysis reported here: (a) whether or not xin was contrasted with the body; and (b) whether it was used to refer to a bodily organ, locus of feelings and emotions (lower cognitive concepts), or a locus of deliberate, reflective cognition (higher cognitive concepts). Next, 620 randomly sampled passages (179 from the Pre, 35 from the Early, and 406 from the Late Warring States period) were each subjected to these 29 dichotomous coding decisions. First, each passage (presented in a random order) was independently coded by two of three coders; that is, the passages were split among the three possible pairs. Cohen's Kappa reliability levels for these three pairs were .71, .56 and .47. Passages for which both coders' decisions agreed on all 29 codes were considered finalized at this point (310 passages); for the remaining passages coders disagreed on at least one $($ mean $=1.87, S D=1.11)$ coding decision. To resolve these disagreements, a third coder (i.e., the one not in the pair who initially coded that passage) independently coded these passages, and where their 29 decisions corresponded exactly to one of the first two coders, these passages were again considered finalized (159 passages). The disagreements on the remaining (151) passages were arbitrated and finalized by the first author, an expert on pre-Qin Chinese texts with full access to the original coders' decisions and notes. ${ }^{5}$

To provide a baseline for testing the frequency with which xin was explicitly contrasted with the body, ${ }^{6}$ we also coded this contrast for four other commonly-mentioned organs, two external ( $m u$ 目 “eye”, and er 耳 “ear”) and two internal (gan 肝 “liver” and fu 腹 “"stomach"). Of the 864 passages containing occurrences of these terms in the pre-Qin textual database, only 337 also contained one of the predominant "body" terms (xing 形, shen 身, $t i$ 體) and thus were likely candidates for a contrast. These 337 passages were coded by two coders working independently on mutually exclusive subsets. Only one contrast —a single passage where the stomach is contrasted with the body—-was found.

\section{Results}

\subsection{Do early Chinese authors explicitly contrast xin with the body?}

Table 1 shows the frequency of passages that contrast xin with the body in each of the three periods. Since any deviation from zero is statistically significant under a binomial test of the 
Table 1

Frequency in the pre-, early- and late-Warring States periods of three trends

\begin{tabular}{lcccc}
\hline & Pre & Early & Late & All Periods \\
\hline $\begin{array}{l}\text { Contrast with body } \\
\quad \text { Xin-body contrasts }\end{array}$ & $\frac{7}{179}=0.04$ & $\frac{3}{35}=0.09$ & $\frac{42}{406}=0.10$ & $\frac{52}{620}=0.08$ \\
$\begin{array}{l}\text { Semantic reference } \\
\text { b }\end{array}$ & $\frac{8}{179}=0.04$ & $\frac{0}{35}=0.00$ & $\frac{9}{406}=0.02$ & $\frac{17}{620}=0.03$ \\
$\quad$ Bodily organ & $\frac{72}{179}=0.40$ & $\frac{4}{35}=0.11$ & $\frac{53}{406}=0.13$ & $\frac{129}{620}=0.21$ \\
Locus of emotion & $\frac{90}{179}=0.50$ & $\frac{28}{35}=0.80$ & $\frac{324}{406}=0.80$ & $\frac{129}{620}=0.71$ \\
$\quad$ Locus of cognition & $* *$ & $p=.12$ & $* *$ & $* *$ \\
Rates of semantic reference compared & & $* *$ & $* *$ & $* *$ \\
Emotions > Organ & $* *$ & $* *$ & $* *$ & $* *$ \\
Cognition > Organ & $p=.07$ & & & \\
Cognition > Emotions & & & & \\
\hline
\end{tabular}

${ }^{\mathrm{a}}$ Frequency of contrasts between $x$ in and the body.

${ }^{\mathrm{b}}$ Frequency of usage of $x$ in to refer to a bodily organ, the locus of emotions or the locus of cognition.

${ }^{\mathrm{c}}$ Results of $\chi^{2}$ significance tests of differences in these frequencies. Note that a conservative Bonferoni-Sidak adjusted alpha level for this many tests is approximately .004.

$* * p<<.004$.

hypothesis $p=0$, a stronger test of this hypothesis is to compare these rates to the frequency with which other organs are contrasted with the body. A simple logistic regression on the 1/864 non-xin passages and 52/620 xin passages that contrast an organ with the body shows that the odds of xin being contrasted with the body were about 77 times greater than the other organs we examined $(p<<.001)$.

\subsection{Did the frequency of such contrasts increase with time?}

We used logistic regression to model the dichotomous outcome of whether xin was contrasted with the body in each passage, predicted by the period in which the passage was written. ${ }^{7}$ The frequency of contrasts did increase with time: The odds of xin-body contrasts were approximately 2.3 times $(p=.24)$ higher in the Early than Pre period, 2.8 times $(p=.01)$ higher in the Late than Pre period and 1.2 times $(p=.74)$ higher in the Late than Early period.

\subsection{Does xin 心 refer to a physical organ, locus of higher cognition ${ }^{8}$ or locus of emotion?} Does its reference change with time?

To compare the frequency with which xin referred to each of primary content codes, ${ }^{9}$ we again fit our dichotomous data to a logistic regression model. The relative rates of occurrence of these three references are presented in Table 1. The frequency with which xin referred to body did not differ significantly between the three periods $\left(\chi^{2}=2.24, p=.33\right)$, but the rates of reference to $x i n$ as locus of higher cognition $\left(\chi^{2}=51.07, p<.01\right)$ and 
emotion $\left(\chi^{2}=52.69, p<.01\right)$ did. Locus of higher cognition was referred to significantly more in the Early (odds-ratio $=3.96, p<.01$ ) and Late (odds-ratio $=3.91, p<.01$ ) periods than in the Pre period, but did not significantly differ between the Early and Late periods $(p=.98)$. Meanwhile, xin as locus of emotion showed the reverse pattern: It was referred to significantly less in the Early (odds-ratio $=0.19, p<.01$ ) and Late (odds-ratio $=0.22$, $p<.01)$ periods than the Pre period, while also not significantly differing between the Early and Late period $(p=.78)$.

A perhaps simpler way to analyze this trend is to approximate temporal periods linearly (i.e., Pre $=0$, Early $=1$, Late $=2$ ), which shows that the rate of $x$ in's reference to the locus of cognition increased with time $(\beta=0.67, p<.01)$ and its reference to the locus of emotion decreased with time $(\beta=-0.75, p<.01)$, while the linear change in rate of reference to a bodily organ did not differ significantly from chance $(\beta=-0.36, p=.17)$ despite our considerable sample size. The interaction terms describing the differences between these rates were highly significant for contrasts between cognition versus emotion, as well as cognition versus bodily organ $(p<.01)$, while the changes in rates of reference to emotion versus bodily organ did not differ significantly from chance $(p=.18)$.

To test whether the rates of reference within each period differed more than expected by random sampling alone, we conducted $\chi^{2}$ tests of the difference between these proportions given binomial expectations. ${ }^{10}$ Even given conservative Bonferonni-Sidak adjusted alpha levels, all but two of these proportions were significantly different (confidence interval overlaps in Fig. 1 make this visually apparent), the exceptions being xin referring to a locus of

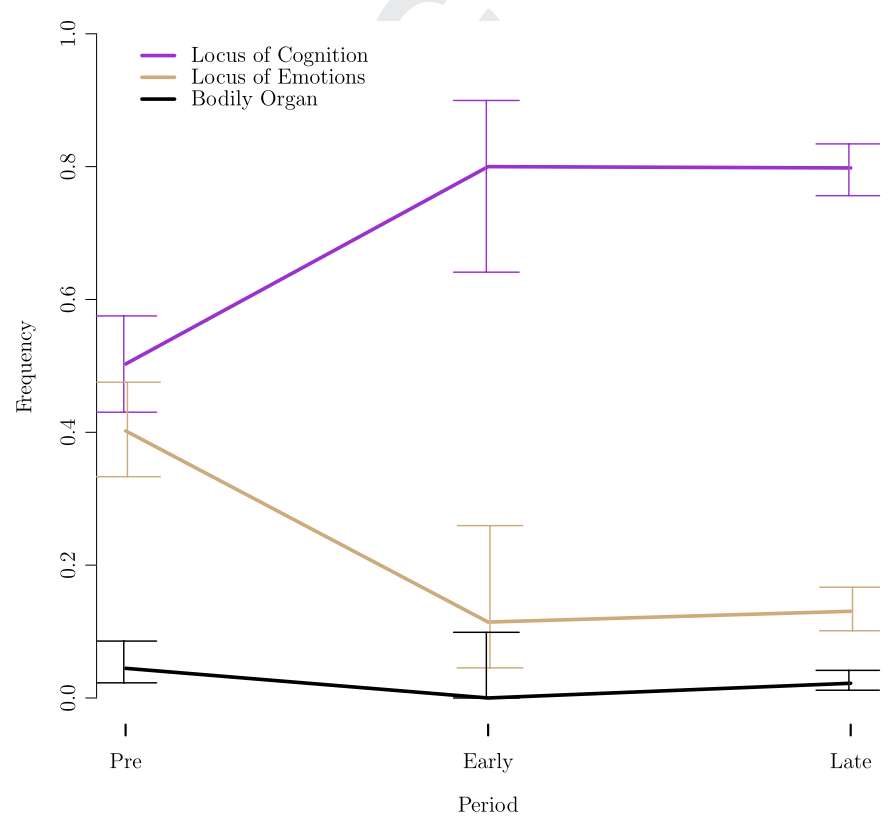

Fig. 1. Temporal trends in the rate at which xin refers to a bodily organ, the locus of emotions or the locus of cognition, in the pre-,early- and late-Warring States periods, with 95\% confidence intervals. 
both emotions and cognition at similar rates in the Pre period $(p=.07)$ and to a physical organ and locus of emotions at similar rates in the Early period $(p=.1)$; see Table 1 .

The general pattern of these findings is illustrated in Fig. 1. Throughout all three periods xin referred to a physical body organ at a consistently low rate (about 3\%). During the Pre Warring States period, it referred about equally often to a locus of emotion (approx. 40\%) or cognition (approx. 50\%). By the Early period it was being used to refer to the locus of cognition far more frequently (approx. 80\%) than emotions (approx. 10\%), and this pattern persisted into the Late period. This change also corresponded to a rise in the frequency of explicit contrasts of xin with the physical body.

\section{Discussion}

Our large-scale quantitative textual analysis found a clear pattern in the historical use and reference of the Chinese word xin (heart/heart-mind). Though it refers to a physical organ in the body (the heart) at a consistently low rate $(17 / 620 \approx 3 \%)$ throughout the time periods analyzed, it was explicitly contrasted with the body at a higher $(52 / 620 \approx 8 \%)$, statistically greater $(p<.01)$ rate. Moreover, it alone of the all the organs is regularly contrasted with the common terms that refer to the physical body (xing 形, shen 身, $t i$ 體). In addition, although xin is often portrayed as the locus of emotion as well as other cognitive abilities in the Pre Warring States period, by the end of the Warring States there is a strikingly clear trend whereby xin is less and less associated with emotions and becomes increasingly portrayed as the unique locus of "higher' cognitive abilities (which follows from WFD, but not SFD). The fact that this conception of xin then fixates for the remaining 2000 years of Chinese history argues against seeing this shift as the sort of random variation consistent with either $\mathrm{MBH}$ or cultural constructivist accounts.

What is so interesting about the early Chinese case is that linguistic resources seem to militate against mind-body dualism: The term that came to refer to mind concepts was represented by a graph denoting the physical heart, a concrete organ embedded in the body and the locus of desires and emotions. Nonetheless, over a several hundred year period, semantic distinctions in this culture became increasingly split along lines that strikingly mirror modern Western folk conceptions of body and mind, and that remain the default picture for the rest of its history. ${ }^{11}$

Although likely predated by short inscriptions on divinatory bones and ceremonial bronze vessels, the pre-Warring States materials surveyed constitute the first significant writing from China, and date from a period of relative social, political, and religious stability, with literacy confined to an extremely small class of specialists. The Warring States period-especially its latter half-represents a significant rupture with the past, with the central polity fragmenting into a variety of independent states, all vying for supremacy. The chaos of the Warring States_also known as the period of "The Hundred Schools" — was accompanied by a dramatic expansion in literacy and a profusion of opposing schools of thought, each peddling its own particular model of self-cultivation and politico-religious 
thought to rulers and advisors desperate for ideas that would give them a competitive advantage. We speculate that the concept of xin that we see in pre-Warring States materials represents a relatively undeveloped, and therefore undifferentiated, concept that was suddenly and rapidly elaborated over the course of the Warring States Period. Discussion of the causes of this semantic shift is necessarily speculative. The possibility that we find most plausible and directly consistent with our evidence is that the evolution of xin was guided by the universal cognitive division proposed by WFD - that is, the referent of this particular written character was attracted to a shared cognitive default in response to its use by a rapidly growing literate community.

One alternative hypothesis is a cultural evolutionary one: That is, that Pre Warring States Chinese were holists, but learned and subsequently culturally transmitted the useful cognitive trick of dualism. Our corpus analysis techniques cannot distinguish between this hypothesis and our favored cognitive default hypothesis, but the latter is much more consistent with contemporary experimental evidence suggesting that mind-body dualism arises quite early in childhood development (reviewed in Bloom, 2004). Another alternative is the genre hypothesis: Approximately 38\% of the Pre Warring States material is lyric poetry-a genre more or less absent from Early and Late material-which might be expected to include a disproportionate number of references to emotions. Rigorous analysis of postWarring States material remains to be done, but preliminary examination suggests that the reemergence of lyric poetry in the later corpus is not accompanied by a reversion in the conception of xin to the Pre Warring States pattern. The relative invariance of the conception of xin in post-Warring States times also argues against an audience-effect hypothesis, whereby the shift in xin's referent is a response to the particular rhetorical needs of Early and Late Warring States thinkers, in which case one would expect further shifts in reference over time.

A complete test of dualism would require extensive comparison of many historical and contemporary cultures; we hope that the methodologies pioneered in this study might serve as a template for work that will expand the scope of historical cross-cultural work on concepts important to cognitive scientists. Nonetheless, our analysis of pre-Qin China represents an important contribution to this puzzle. Predating the long-range, large-scale exchange of goods and ideas along the Silk Road that began in the early Common Era, pre-Qin China represents a culture, tied together by a common writing system, that developed in conceptual isolation from other literate world cultures. As such, it represents a unique and influential data point in human cultural phylogeny, which can be of great value in disentangling cultural, ecological, and evolutionary influences on the modern cognitive differences being catalogued by cultural psychologists (e.g., Heine \& Hamamura, 2007; Nisbett, 2003).

Moreover, pre-Qin China-a time of great intellectual foment, and the formative period of Chinese religious thought - has left to us a rich and well-documented archeological heritage, as well as massive and fascinating corpus of received texts in a variety of genres. This received corpus is now being supplemented with an increasingly large collection of archeological texts that represent a direct transmission from pre-Qin China to the present, eliminating concerns about later scribal errors or editorial changes. For scholars interested in human 
cognitive universals, evidence from pre-Qin China should be viewed as an ideal, and essential, data point.

Besides its implications for universalist claims concerning folk dualism, our study also serves as an example of how techniques from the sciences-large-scale, team-based analysis, random sampling, statistical analysis - can be put to good use in the humanities. We believe that the approach developed in this study can be easily adapted for use in accessing other historical materials in order to address, in a rigorous and quantifiable manner, a wide variety of questions that interest cognitive scientists. Acquiring "data from dead minds" 12 in this fashion has its limits: We cannot perform controlled experiments on our ancient Chinese subjects, and it is uncertain how directly written materials produced by a small, literate elite are related to either everyday implicit cognition or popular conceptions. On the other hand, such data do tell us something about human cognition in cultures far removed in time, and therefore helps to expand the scope of cognitive science research beyond its standard - and likely rather unrepresentative-Western undergraduate subject pool (Henrich, Heine, \& Norenzayan, 2010).

\section{Notes}

1. See, for instance, Brashier, 1996 on multipartite views of the soul in early China, as well as work by Richert and Harris that has challenged Bloom by arguing that a tripartite (body-mind-soul) model of the self is more characteristic of folk thinking than simple mind-body dualism (Richert \& Harris, 2008). This apparent refutation of Bloom is converted into a corrective when one observes that nothing that does not have a mind can possess a soul, which suggests that soul concepts - unitary or multipartite - are simply subdivisions of the more basic category of mind.

2. Kokoro is the Japanese pronunciation of the character xin 心 that is the focus of the study reported below.

3. An on-line database maintained by the National Palace Museum in Taipei, Taiwan (http://210.69.170.100/s25/index.htm).

4. An on-line database of the Guodian corpus of bamboo texts (interred roughly 300 BCE and discovered in 1993), maintained by the Chinese University of Hong Kong (http://bamboo.lib.cuhk.edu.hk/).

5. In order to control for the possibility of bias having been introduced in the final round of coding, we repeated the analyses reported below using only those passages finalized in the first two rounds by agreement between at least two independent coders. We found a qualitatively identical pattern of results, specifically: All significance levels remained on the same side of conventional thresholds (i.e., .05, .01 and .001), all effects retained their directions, and their magnitudes remained close to those reported.

6. Instances of xing 形, shen 身, $t i$ 體, $l i$ 力 (physical strength) and $q i$ 氣 (when used in the sense of physiological energy) were all taken as references to the "body." 
7. Note that this statistical procedure examines differences in the odds of a contrast, not the absolute number of contrasts.

8. Note that, for the purposes of the final analysis, content codes 3-5 (all referring to various aspects of what one might term "higher cognition") were collapsed into one code.

9. For examples from the texts examined, see the on-line Supporting Information, which also includes our entire coded data set.

10. Hypothesis tests were based on simple asymptotic assumptions, implemented by the prop.test() function of the $\mathrm{R}$ statistical computing environment (version 2.11.1). Confidence intervals in Fig. 1 were computed using Wilson's score method, which has better coverage (Newcombe, 1998).

11. Qualitative analysis suggests that, once Buddhism is introduced to China in the beginning of the Common Era, the conception of xin becomes, if anything, even more disengaged from the body (e.g., Radich, unpublished data).

12. The title of a session organized by Roger Beck and Luther Martin at the International Association for the History of Religion $\mathrm{XX}^{\text {th }}$ Quinquennial World Congress, Toronto, ON, August 2010.

\section{Acknowledgments}

This project was funded primarily by a grant from Oxford's Cognition, Religion and Theology Project, with additional support from the Canada Research Chairs program and a Social Sciences and Humanities Research Council of Canada (SSHRC) Standard Research Grant awarded to the first author. The authors would like to acknowledge the crucial support of Natalie Henrich, Joe Henrich, Ara Norenzayan and Steve Heine in the early stages of project design, as well as the efforts of our coders, Clayton Ashton, Abraham de Jesus, and Jennifer Lundin Ritchie. Our preliminary results were presented at Oxford University, the International Association for the History of Religion XXth Quinquennial World Congress, and the Southern California Consortium for Cognitive Science of Religion workshop, and we benefitted greatly from audience feedback, especially that of Ray Paloutzian, Jonathan Schooler and Kevin McCulloch. We would also like to thank the anonymous reviewers at Cognitive Science for comments that significantly strengthened the paper.

\section{References}

Bloom, P. (2004). Descartes' baby: How the science of child development explains what makes us human. New York: Basic Books.

Brashier, K. E. (1996). Han thanatology and the division of "souls". Early China, 21, 125-158.

Cohen, E. (2007). The mind possessed: The cognition of spirit possession in an Afro-Brazilian religious tradition. Oxford, England: Oxford University Press.

Cohen, E., \& Barrett, J. (2008). When minds migrate: Conceptualizing spirit possession. Journal of Cognition and Culture, 8, 23-48. 
Geaney, J. (2002). On the epistemology of the senses in early Chinese thought. Honolulu, HI: University of Hawaii Press.

Granet, M. (1934). La pensée chinoise. Paris: La Renaissance du livre.

Heine, S. J., \& Hamamura, T. (2007). In search of East Asian self-enhancement. Personality and Social Psychology Review, 11, 1-24.

Henrich, J., Heine, S. J., \& Norenzayan, A. (2010). The weirdest people in the world? Behavioral and Brain Sciences, 33(2-3), 61-83.

Hodge, K. M. (2008). Descartes' mistake: How afterlife beliefs challenge the assumption that humans are intuitive Cartesian substance dualists. Journal of Cognition and Culture, 8, 387-415.

Jullien, F. (2007). Vital nourishment: Departing from happiness. Translated by A. Goldhammer. New York: Zone Books.

Lévy-Bruhl, L. (1922). La mentalité primitive. Paris: Alcan.

Lewis, M. E. (1999). Writing and authority in Early China. Albany, NY: SUNY Press.

Newcombe, R. (1998). Interval estimation for the difference between independent proportions: Comparison of eleven methods. Statistics in Medicine, 17(8), 873-890.

Nisbett, R. (2003). The geography of thought. New York: Free Press.

Richert, R. A., \& Harris, P. L. (2008). Dualism revisited: Body vs. Mind vs. Soul. Journal of Cognition and Culture, 8, 99-115.

Rosemont, H. Jr, \& Ames, R. (2009). The Chinese classic of family reverence. Honolulu: University of Hawai'i Press.

Wierzbicka, A. (2006). On folk conceptions of mind, agency and morality. Journal of Cognition and Culture, 6, $165-179$.

Yu, N. (2007). Heart and cognition in ancient Chinese philosophy. Journal of Cognition and Culture, 7, 27-47.

\section{Supporting Information}

Additional Supporting Information may be found in the online version of this article on Wiley Online Library:

Table S1. Dichotomous criteria used to code passages containing xin. The first two main sets of codes (labeled contrasts and content) were used directly in the analyses reported here; the remaining codes are relevant for interrogating other research questions and for understanding the coding procedure and reliability measures.

Please note: Wiley-Blackwell is not responsible for the content or functionality of any supporting materials supplied by the authors. Any queries (other than missing material) should be directed to the corresponding author for the article. 